\title{
Opportunistic Network Coding and Signal Combining ARQ for Two-Way DF Relay Network
}

\author{
Fei Wang ${ }^{1, a^{*} \text {, Dongmei Zhang }}{ }^{2, b}$, Kui $\mathrm{Xu}^{3, \mathrm{c}}$, Jingzhou Yang ${ }^{4, \mathrm{~d}}$, Yang Liu ${ }^{5, \mathrm{e}}$ \\ ${ }^{1,2,3,4}$ College of Communications Engineering, PLA University of Science and Technology \\ Nanjing 210007, China \\ ${ }^{5}$ Unit 73667 of PLA, Nanjing 211132, China \\ awfzd01@sina.com, ${ }^{b} z$ hangdm72@126.com, ${ }^{\mathrm{C}}$ Igdxxukui@126.com
}

\begin{abstract}
Keywords: Two-way DF relay, buffer-aided relaying, opportunistic network coding, signal combining.

Abstract. For the purpose of improving the throughput and reliability of the two way decode-and-forward (DF) relay networks, we propose an opportunistic network coding and signal combining (ONCSC) based automatic repeat request (ARQ) protocol. We assume that the relay node (RN) has a buffer which can store the signals received. Hence, RN can combine the signals received from different time slots and judge to transmit or receive in the next time slot according to the output of the maximal likelihood (ML) detector. Two signal combining methods at the RN, namely maximal sum signal-to-noise ratio combining (MSSNR) and maximal minimum signal-to-noise ratio combining (MM-SNR) are proposed in this paper. Simulation results show that the proposed two signal combining methods outperform traditional No-ARQ and No-combining ARQ systems on the packets error ratio (PER) and throughput performances in the moderate SNR. Also the optimal power allocation factor between the sources and $\mathrm{RN}$ is discussed in the simulations.
\end{abstract}

\section{Introduction}

Relaying has been known as a powerful technique to reduce the transmit power and achieve energy efficient transmission by shortening the distance of each hop. A large number of existing works considered the half-duplex relaying (HDR), where the relay transmits and receives using the orthogonal frequency or time resources [1-4]. However, one main drawback of HDR is its inherent spectral efficiency (SE) loss due to half-duplex constraint.

To solve this problem, the application of network coding (NC) to the two-way relay network (TWRN) has been investigated in [5-9] to improve the SE performance. The relay node first XORs two packets received from different sources and then broadcasts the network coded packet to all the users. Each user can get the expected information simply by XORing the received packet with its own packet. By mixing different packets into one packet, $\mathrm{NC}$ can reduce the number of transmissions and hence leading to higher throughput as well as lower transmission energy consumption $[10,11]$.

Recently, the automatic repeat request (ARQ) scheme is introduced in NC based TWRN system to enhance the link reliability. In this paper, we discuss the applicability of traditional ARQ protocols of Stop-Wait (SW) in the context of NC-based TWRN. Usually, there are two main strategies to be employed at the relay: amplify-and-forward (AF) [12] and decode-and-forward (DF) [6]. In this paper, we focus on the DF strategy as the noise can also be amplified for AF compared to DF except in very high SNR, which can severely degrade the performance of the system. In addition, the relay can decode the received packets and thus have a chance to decide whether to retransmit or not in the next time slot.

Three ARQ protocols are proposed in [13] to improve the link reliability and throughput for DF based TWRN. In [14], the throughput of a TWRN ARQ system with and without the direct link between two users is compared. In [15], two improved NC-based ARQ schemes are investigated to increase the system throughput for TWRN. A cooperative ARQ method with network coding is proposed for TWRN in [16], and the binary Markov channel is applied which takes the correlation of the channel coefficients in time domain into account. The buffer-aided TWRN was also investigated in 
$[17,18]$, wherein the DF method is adopted and RN switches between different working modes adaptively to maximize the system throughput. The signal combining strategies at the RN in a ARQ based DF TWRN system have not been investigated so far. In this paper, we assume that the RN has a buffer which can store and combine the signals received in different time slots. Two signal combining methods at the RN, namely maximal sum signal-to-noise ratio combining (MSSNR) scheme and maximal minimum signal-to-noise ratio combining (MM-SNR) scheme are presented to improve the reliability of the system.

For comparison purpose, we analyze the performance of our proposed approach in terms of PER and throughput performance. Simulation results show that the performance of the proposed schemes is better than traditional No-ARQ scheme [19] and buffer-aided No-combining ARQ two-way relaying $[17,18]$ scheme.

The remainder of this paper is organized as follows. Section II describes the system model and ARQ strategy. The combining approach of the relay is proposed in Section III. Section IV shows some simulation results. Moreover, we conclude this paper in Section V.

\section{System Model}

Fig. 1 presents the system model of the DF based TWRN considered in this paper, two single-antenna users $\mathrm{T} 1$ and $\mathrm{T} 2$ exchange information assisted by $\mathrm{R}$ and we assume that there is no direct link between the two users. All nodes are work in half-duplex mode, i.e. a node cannot simultaneously transmit and receive packets. Although the TWRN is simple, it is a fundamental structure of a wireless network, and operations of the relay node in a TWRN are similar to that in a complicated wireless network.

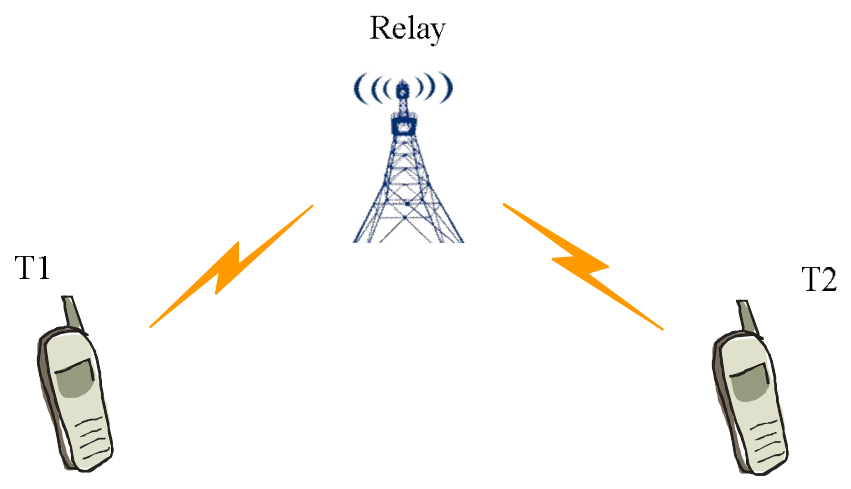

Fig. 1. A two-way relay network.

All the wireless links are assumed to be flat fading Rayleigh channels with independent identically distributed (i.i.d) and all the channel coefficients are constant during one time slot and change independently from one slot to another. The channels are assumed to be reciprocal as time-division-duplex method is used. The perfect instantaneous channel state information can be obtained by all the receivers through the use of training sequences. The transmitted power of the users and the RN are $P_{T 1}, P_{T 2}$ and $P_{R}$. The data exchange procedure consists of two consecutive phases: multiple access (MA) phase and broadcast (BC) phase. Without loss of generality, we assume that all delays and frequency costs generated by ARQ are ignored. Besides, perfect system synchronization is also need.

\section{Proposed Opportunistic Network Coding ARQ Strategy}

The transmission process of the proposed opportunistic network coding ARQ strategy can be described as follows:

In the MA phase: $\mathrm{T} 1$ and $\mathrm{T} 2$ transmit the signal $x_{1}$ and $x_{2}$ to $\mathrm{RN}$ at the same time, respectively. The $\mathrm{RN}$ stores the received signal in the buffer. The received signal can be expressed as: 


$$
y_{R}=\sqrt{P_{T 1}} h_{T 1 k} x_{1}+\sqrt{P_{T 2}} h_{T 2 k} x_{2}+n_{R}
$$

where $x_{1}$ and $x_{2}$ are the transmitted symbols by T1 and T2, respectively, $h_{T 2 k}$ and $h_{T 2 k}$ as the channel gains between T1, T2 and R in the $k$-th time slot , $P_{T i}$ represents the transmission power of the user. Likewise, $n_{R}$ is the additive white Gaussian noise(AWGN) at the $\mathrm{RN}$ with zero mean and a power spectral density (PSD) of $N_{0}$.

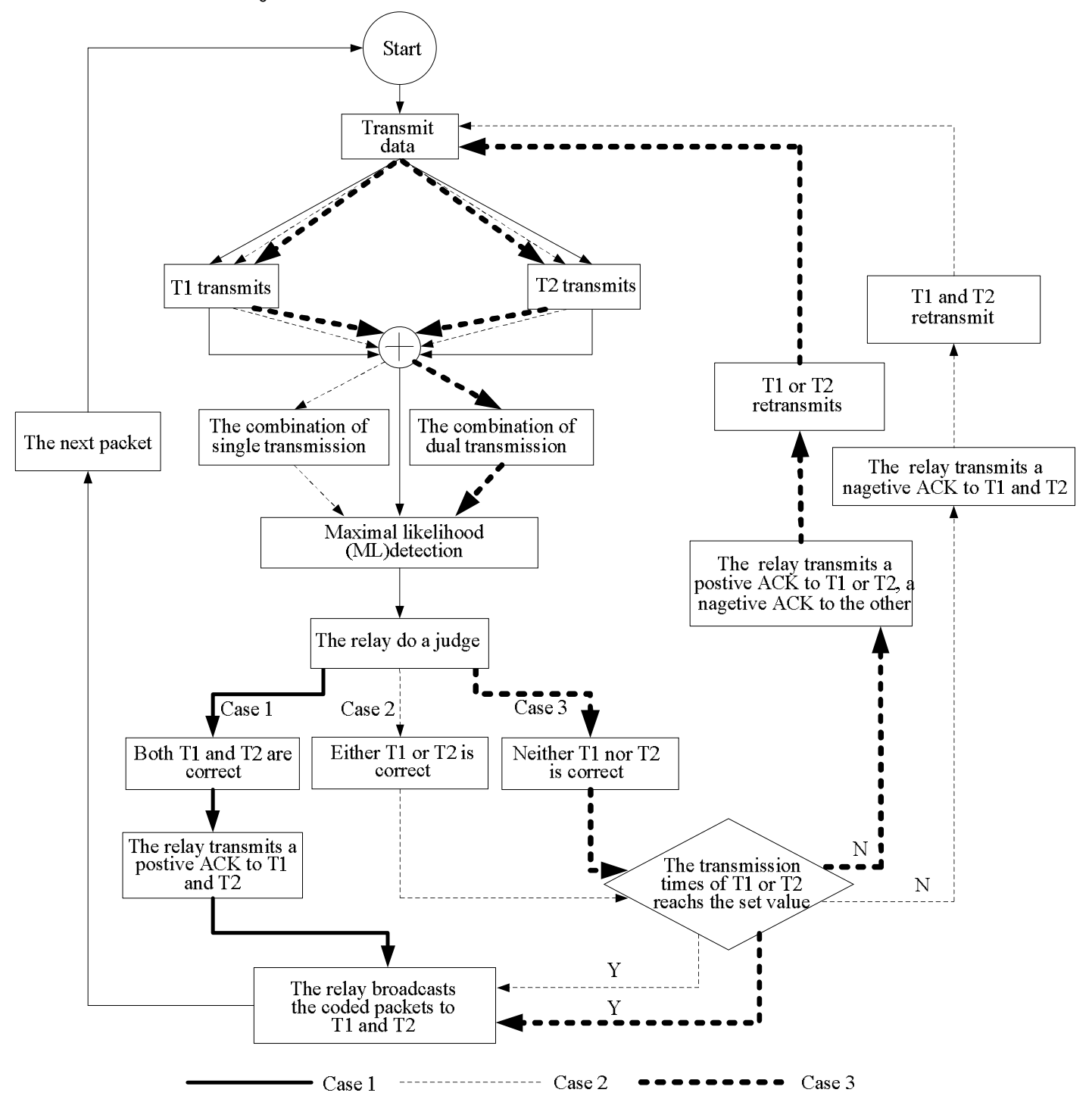

Fig.2. Flow chart of the proposed opportunistic network coding based ARQ strategy.

The RN performs DF strategy by using the following ML detector [20]:

$$
\begin{gathered}
\left(\hat{x}_{1}, \hat{x}_{2}\right)=\operatorname{argmin}\left\{\left\|y_{R}-y_{\text {com }}\right\|^{2}\right\} \\
y_{\text {com }}=\sqrt{P_{T 1}} h_{T 1 k} x_{1}+\sqrt{P_{T 2}} h_{T 2 k} x_{2}
\end{gathered}
$$

Note that, for each packet, besides CRC, the extra information about the corresponding owner should be included [16]. Then, the relay does a judge to receive or transmit in the next slot according to the result of the ML detection. Hence, the proposed opportunistic network coding based ARQ strategy includes three cases:

Case A: Two-way transmission.

If the RN fails to decode both of the two packets, that is $\hat{x}_{1} \neq x_{1}, \hat{x}_{2} \neq x_{2}$, the RN feeds back two negative ACK messages to inform two users to retransmit their packets in the next time slot. After retransmission, the $\mathrm{RN}$ stores the received packet in the buffer. Before the next packet arrived, the RN 
combines the packets stored in the buffer, then, the $\mathrm{RN}$ does a judge to transmit or wait according to the ML result.

Case B: One-way transmission.

If the RN can decode only one of the two packets, that is $\hat{x}_{1}=x_{1}, \hat{x}_{2} \neq x_{2}$, the RN feeds back a positive ACK and a negative ACK to two users. For example, if $\mathrm{x} 1$ can be successfully detected, the RN feeds back a positive ACK and a negative ACK to T1 and T2, respectively. In the following time slot, only $\mathrm{T} 2$ retransmits its packet to $\mathrm{R}$. Then, the RN conducts a judgement according to the output of the ML detector as Case A.

Case C: Network coding transmission.

If the RN can successfully decode both of the two packets with no error, that is $\hat{x}_{1}=x_{1}$ and $\hat{x}_{2}=x_{2}$. The RN don't need to store the received packets. A positive ACK is broadcasted to both of the two users. In the next time slot, the RN will send the network coding combined packet to the two users. The flow chart of the proposed opportunistic network coding based ARQ strategy is given in Fig .2. As shown in this figure, the completion of a round depends on the condition that both packets from two source nodes are successfully decoded by the relay nodes. At the end of the MA phase, if at least one packet fails, retransmission phase starts. During the previous $k-1$ time slots, several copies of the signals are stored in the buffer, the $\mathrm{RN}$ firstly combines them. For the two-way transmission, the signal received by the $\mathrm{RN}$ for the $k-1$ slots can be expressed as:

$$
\mathbf{y}=\sqrt{P_{T 1}} \mathbf{h}_{T 1 k-1} x_{1}+\sqrt{P_{T 2}} \mathbf{h}_{T 2 k-2} x_{2}+\mathbf{n}_{R}
$$

where $\mathbf{h}_{T 1 k-1}=\left[h_{T 11}, \mathrm{~L}, h_{T 1 k-1}\right]^{T}, \mathbf{h}_{T 2 k-1}=\left[h_{T 21}, \mathrm{~L}, h_{T 2 k-1}\right]^{T}$ are the channel vectors. The combined signal can be expressed as:

$$
y^{*}=\mathbf{w}^{H} \mathbf{y}=\sqrt{P_{T 1}} h_{T 1 k-1}^{c o m} x_{1}+\sqrt{P_{T 2}} h_{T 2 k-1}^{c o m} x_{2}+\dddot{N}_{R}
$$

where $h_{T i k-1}^{\text {com }}=\mathbf{w}^{H} \mathbf{h}_{T i k-1}, i=1,2$ are the combined channel gains, $\mathbf{w}$ stands for the combining vector with Frobenius norm $\|\mathbf{w}\|=1$. Likewise, $\mathscr{V}_{R}$ is the equivalent noise after combining. The RN decode $x_{1}, x_{2}$ using $h_{T i k-1}^{\text {com }}, i=1,2$ to decide whether to receive or to transmit in the $k$-th slot.

If the system works in the one-way transmission, for instance, we assume that RN has already successfully decode $x_{1}$ in the $n(n \leq k-1)$ time slot, the T2 needs to retransmit $x_{2}$ to the RN. Similar to the two-way transmission, the $\mathrm{RN}$ combines the received signals and decodes the signal of $\mathrm{T} 2$ to decide whether to transmit or to receive. However, in this case, $\mathbf{h}_{T 1 k-1}$ in (4) should be rewritten as $\mathbf{h}_{T 1 k-1}=\left[h_{T 11}, h_{T 12}, \mathrm{~L}, h_{T 1 n}, 0, \mathrm{~L}, 0\right]^{T}$.

In this paper, we set a maximum number of retransmission of $N$ times for the proposed ARQ scheme. The reliability of the system can be improved by increasing $N$ which on the other hand may decreases the overall throughput of the system as more time slots are used to deliver a packet. In addition, the RN still broadcast the packets if it is not successfully decoded within $N$ times retransmission, thus the whole delay of the proposed ARQ scheme can be controlled within a certain number of time slots.

In the BC phase: The relay maps $\left(\hat{x}_{1}, \hat{x}_{2}\right)$ to $x_{R}, x_{R}=M\left(\hat{x}_{1}, \hat{x}_{2}\right)$, by using the mapping principle $\mathrm{M}[*]$ [6]. R broadcasts the regenerated signal $x_{R}$ to $\mathrm{T} 1$ and $\mathrm{T} 2$. Then the signal received by $\mathrm{T} 1$ and $\mathrm{T} 2$ can be expressed as:

$$
y_{T 1}=\sqrt{P_{R}} h_{T 1 k} x_{R}+n_{1}
$$

and

$$
y_{T 2}=\sqrt{P_{R}} h_{T 2 k} x_{R}+n_{2}
$$

Respectively, where $n_{i}, i=1,2$ is the AWGN at the user with zero mean and PSD of $N_{0}$. Each user decodes the signals of the counterpart using the operation of XORing. Meanwhile, the buffer of the relay is also be wiped for the next round of transmission. 


\section{Signal Combing Approaches At The Relay Node}

Since the received signal-to-noise ratio (SNR) determines the maximum achievable data rate and (essentially) determines the probability of error, hence it is an effective measure of the Quality of Service (QoS). We consider two combining approaches from the view.

The first approach is to maximize the total transmission power, and the second is a "fair" design method in which we attempt to maximize the smallest SNR of the two received signals. Also the optimal combining method for the one-way transmission is considered.

A. Signal combining for the two-way transmission

1) MS-SNR Scheme

In the MS-SNR scheme, the relay node attempts to maximize the sum SNR of the received signals. As $\|\mathbf{w}\|=1$, the PSD of the combined noise is $N_{0}$. The sum power of the signals received in $k$ time slots can be expressed as:

$$
P_{\text {sum }}=P_{R} \mathbf{w}^{H}\left(\mathbf{h}_{T 1 k} \mathbf{h}_{T 1 k}^{H}+\mathbf{h}_{T 2 k} \mathbf{h}_{T 2 k}^{H}\right) \mathbf{w}
$$

By using $\mathbf{H}_{\text {sum }}=\mathbf{h}_{T 1 k} \mathbf{h}_{T 1 k}^{H}+\mathbf{h}_{T 2 k} \mathbf{h}_{T 2 k}^{H}$, which is a positive definite Hermitian matrix, the formulation can be transformed to the following optimization problem:

$$
\begin{aligned}
& \max _{\mathbf{w}} \mathbf{w}^{H} \mathbf{H}_{\text {sum }} \mathbf{w} \\
& \text { s.t. }\|\mathbf{w}\|=1
\end{aligned}
$$

It is shown in [21] that the optimal combining vector $\mathbf{w}$ is the eigenvector corresponding to the maximum eigenvalue of $\mathbf{H}_{\text {sum }} \cdot \mathbf{H}_{\text {sum }}$ can be expressed as $\mathbf{H}_{\text {sum }}=\mathbf{U} \boldsymbol{\Sigma} \mathbf{U}^{H}$, where $\mathbf{U}$ is the square $(k \times k)$ matrix whose $i$-th column is the eigenvector $\mathbf{U}_{i}$ of $\mathbf{H}_{\text {sum }}$ and $\boldsymbol{\Sigma}$ is the diagonal matrix whose diagonal elements are the corresponding eigenvalues $\lambda_{i}$, i.e., $\Sigma_{i, i}=\lambda_{i}$. Thus the optimal $\mathbf{w}$ can be obtained as $\mathbf{w}=\mathbf{U}_{\max }$, where $\mathbf{U}_{\max }$ is the eigenvector associated to the maximum eigenvalue $\lambda_{\max }$ of $\mathbf{H}_{\text {sum }}$.

2) MM-SNR Scheme

We now consider the related problem of maximizing the minimum received SNR. In the MM-SNR scheme, the RN attempts to maximize the minimum SNR of the two received signals. The power of the two combined signals can be written as $P_{i}=P_{T i} \mathbf{w}^{H} \mathbf{H}_{T i} \mathbf{w}, i=1,2$, where $\mathbf{H}_{T i}=\mathbf{h}_{T i k} \mathbf{h}_{T i k}^{H}$.

Thus the SNRs of the two signals at the relay node are $\mathrm{SNR}_{\mathrm{Ti}}=\mathrm{P}_{\mathrm{Ti}} / \mathrm{N}_{0} \mathbf{w}^{H} \mathbf{H}_{T i} \mathbf{w}$. The MM-SNR scheme can be expressed as:

$$
\begin{gathered}
\max _{\mathbf{w}} \min _{i}\left\{\left|\mathbf{w}^{H} \mathbf{h}_{T i k}\right|^{2}\right\} i=1,2 \\
\text { s.t. }\|\mathbf{w}\|=1 .
\end{gathered}
$$

Problem (9) is equivalent to the following problem:

$$
\begin{gathered}
\max _{\mathbf{w}} \min _{i}\left\{\left|\mathbf{w}^{H} \mathbf{h}_{T i k}\right|^{2}\right\} i=1,2 \\
\text { s.t. }\|\mathbf{w}\| \leq 1 .
\end{gathered}
$$

Problem (10) is a non-convex problem which can be solved by adapting a semidefinite relaxation (SDR) approach [22]. On the other hand, we can apply SeDuMi [23] to solve the relaxation problem for the SDR method, and we need to turn (10) into real-valued by the transform formulation (11).In the formulation (11), the symbol $\mathfrak{R}\{\cdot\}$ represents the real part of complex number and $\mathfrak{I}\{\cdot\}$ represents the imaginary part. 


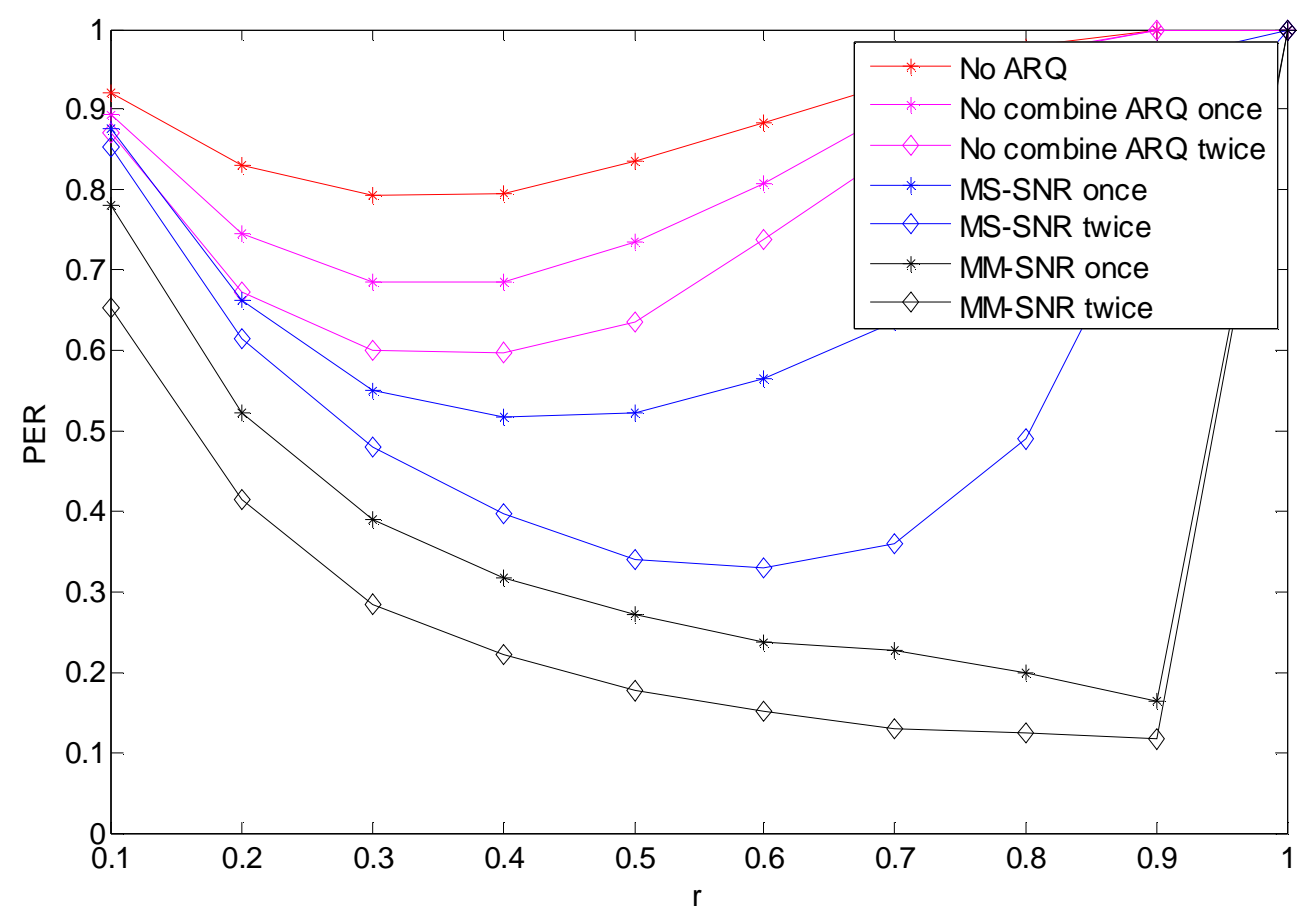

Fig.3. PER as a function of the variable $r$ for the SNR of $25 \mathrm{~dB}$.

$$
\mathbf{H}=\left[\begin{array}{cc}
\mathfrak{R}\{\mathbf{h}\} & -\mathfrak{I}\{\mathbf{h}\} \\
\mathfrak{I}\{\mathbf{h}\} & \mathfrak{R}\{\mathbf{h}\}
\end{array}\right] \Re\{\cdot\}
$$

B. Combine for the one-way transmission

If one of the transmitted signals is successfully detected by the RN, then the system works in the one-way transmission mode. We assume that $\mathrm{RN}$ correctly decodes $x_{1}$, then only $\mathrm{T} 2$ needs to retransmit its signal to the $\mathrm{RN}$. The optimal combining vector $\mathbf{w}$ of the $\mathrm{RN}$ can be obtained by maximizing the received SNR of T2, i.e.,

$$
\begin{gathered}
\max _{\mathbf{w}} \mathbf{w}^{H} \mathbf{H}_{T 2} \mathbf{w} \\
\text { s.t. }\|\mathbf{w}\|=1
\end{gathered}
$$

Just as (8), the optimal $\mathbf{w}$ is the eigenvector associated to the maximum eigenvalue of $\mathbf{H}_{T 2}$.

\section{Numerical Results}

In this section, the average PER and effective throughput performances of the proposed ARQ protocols are analyzed by simulation. Also the corresponding performances of No-ARQ system and No-combining ARQ system are provided for comparisons. We assume that the packets are transmitted by using BPSK modulation for simplicity and the packet length is 256 bits. Throughout simulations, we use the pathloss model $\lambda=d^{-4}$, where $\lambda$ is the path loss coefficient and $d$ is the distance (normalized to 1). As the performance metric, effective throughput is defined as the average number of successfully delivered packets per time unit for each frame, i.e.,

$$
\eta=\frac{E[M]}{E[T]}
$$

where $M$ and $T$ are the total number of successively delivered packets and the total time units to exchange $K$ packets between T1 and T2. As a result, the effective throughput is always bounded by $0 \leq \eta \leq 1$. 


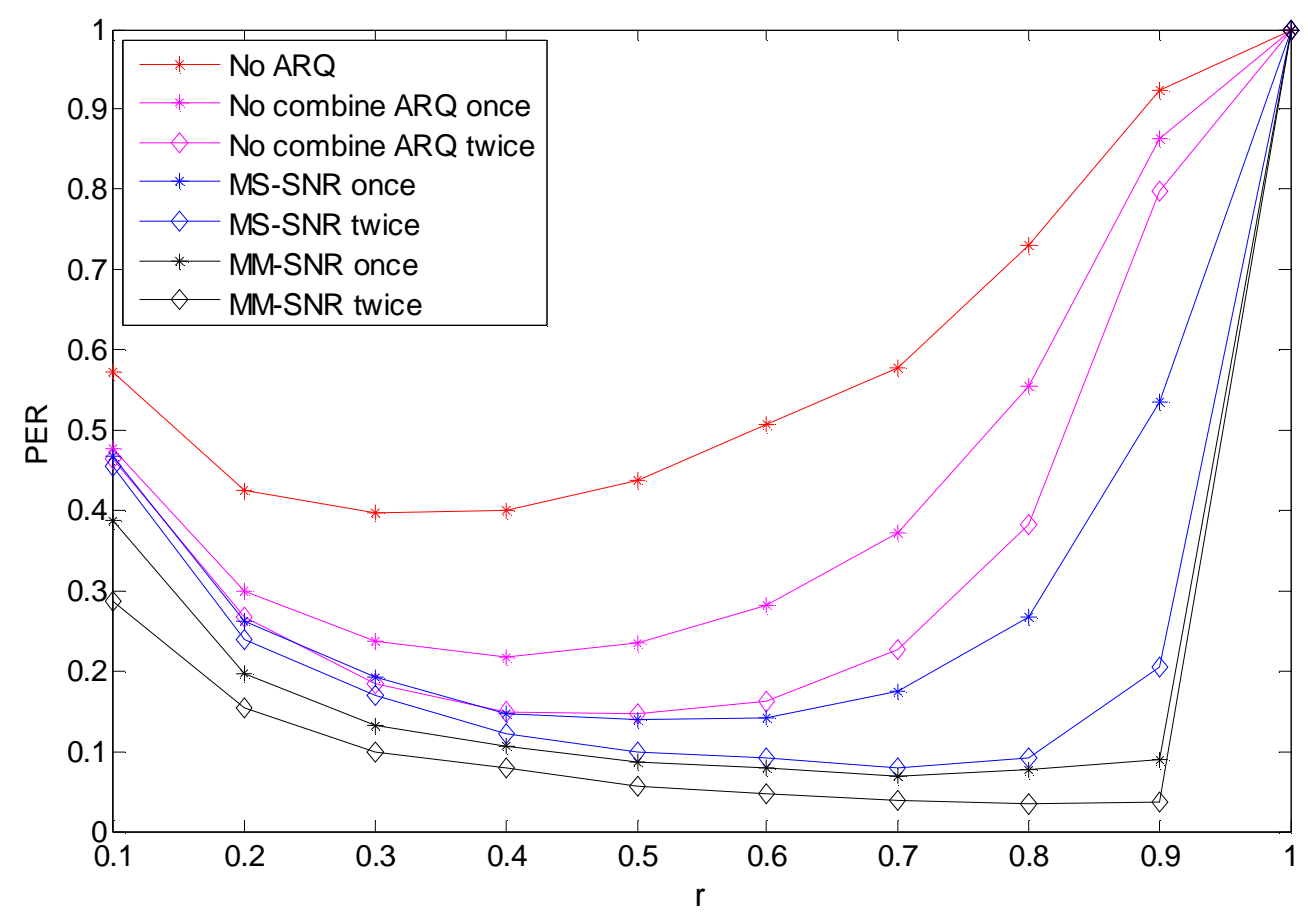

Fig.4. PER as a function of the variable $r$ for the SNR of $30 \mathrm{~dB}$.

Firstly, we are particularly interested in how the PER varies as a function of the power allocation factor $r=P_{R} /\left(P_{R}+P_{T 1}+P_{T 2}\right)$ for the relay at specific SNR values. In Fig 3 and4, PER of the system are plotted as function of $r$, with the No-ARQ, No-combining ARQ and the two combine methods, respectively. On each curve with a given specific SNR value, it is immediately seen that the PER first drop within low $r$ range and reaches a lowest point, then it begins to increase once the factor $r$ is increased beyond the optimal $r^{*}$ which minimizes the PER. We apply the approximate optimal value of $r=0.4$ in the following simulations. That is to say, more power should be allocated to the two users.

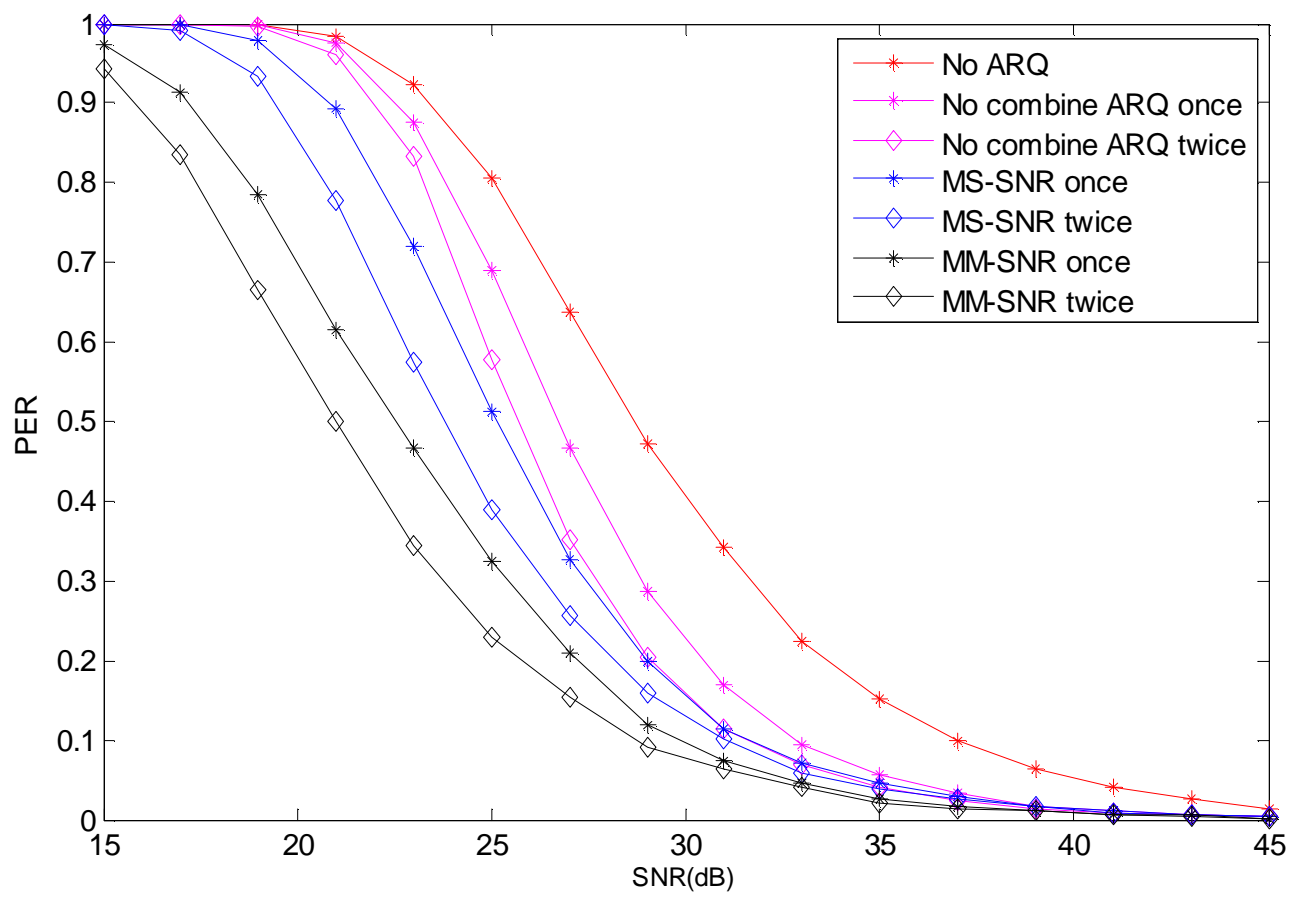

Fig.5. The PER of the proposed ARQ schemes for different SNR. 
Fig 5 shows the PER performance of the proposed opportunistic network coding and signal combining ARQ scheme. At low SNR, all the schemes experience poor performance and the system PER is approximate to 1 , due to the fact that the joint decoding at the relay becomes critical, so we give the curse start from $15 \mathrm{~dB}$ of the SNR for a visual images. The performance gains of the combining schemes are illustrated by comparing with the No-ARQ system and No-combining ARQ system. In the proposed ARQ scheme without relay combining, the RN releases the packets received in the previous slots and only stores the packet received in the current slot. As there is only one packet in the buffer, no combining is performed. It can be observed that the MS-SNR scheme achieves more gain compared with the system without combination at the RN. The MM-SNR scheme outperforms the MS-SNR scheme by about $2 \mathrm{~dB}$ for the once retransmission as the SDR approach is used, but at the cost of high complexity.

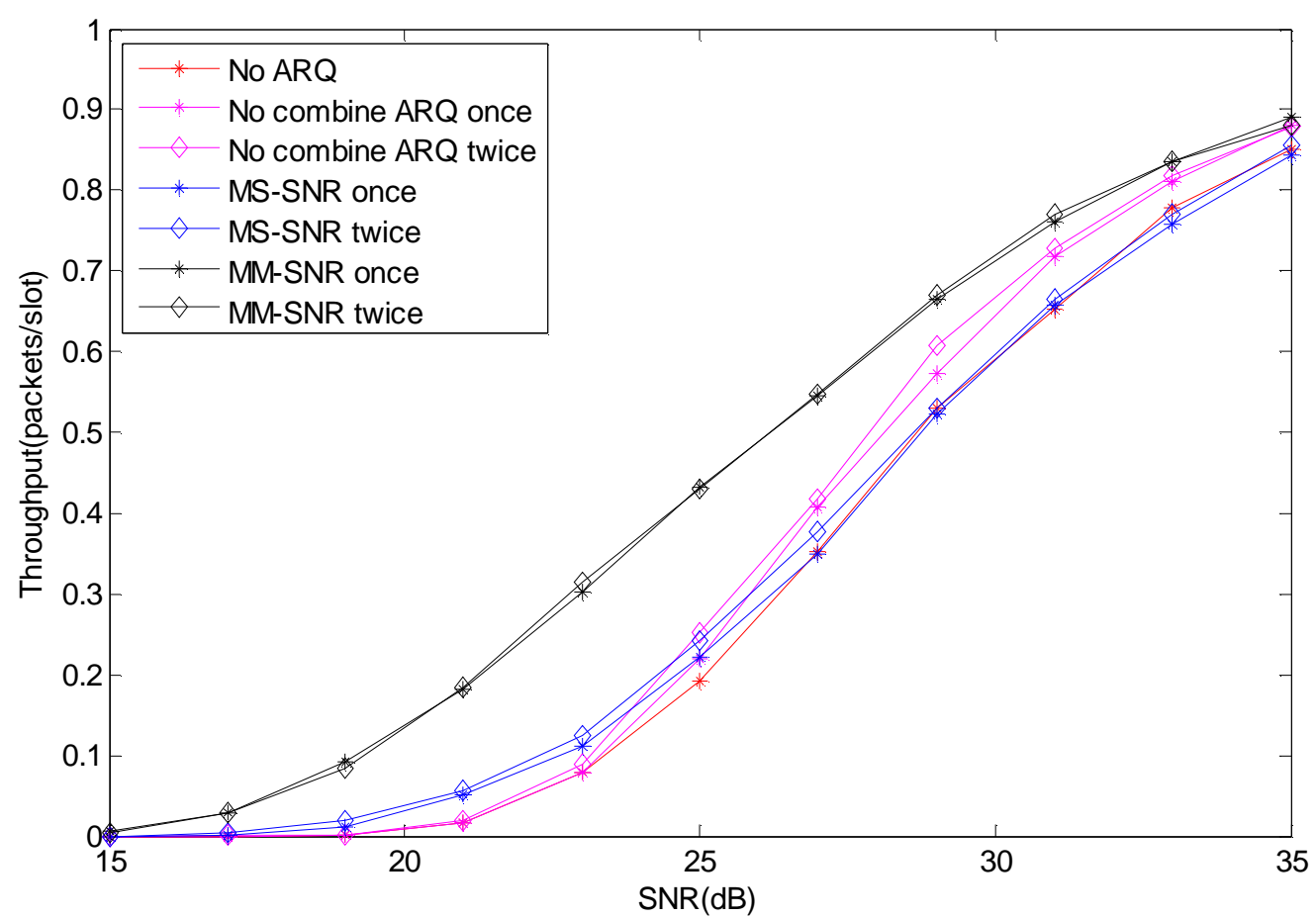

Fig.6. The effective throughput of the proposed ARQ schemes for different SNR.

At last, we simulate the effective throughput of the whole system. The result is as Fig 6. All the effective throughput at low SNR (lower than 15dB) is approximate zero. This is because both the link between T1, T2 and R are so weak that few packets can be decoded correctly in the MA phase. It can be seen from the figure that the MS-SNR method can offer a smoother increase of the throughput curve in moderate SNR (15 dB to $27 \mathrm{~dB}$ ), but a little worse throughput in high SNR. For the MM-SNR method, it can significantly improve the throughput performance with respect to No-ARQ and No-combining ARQ system in two-way relay systems.

\section{Conclusion}

In this paper, we propose an opportunistic network coding and signal combining ARQ for DF TWRN. Two signal combining schemes, i.e., MS-SNR scheme and MM-SNR scheme are studied to further improve the PER and throughput efficiency performances. The best strategy in the sense of PER is the MM-SNR scheme at the cost of a high computational complexity by using SDR. The PER performance of the two schemes performs better than traditional No-ARQ system and No-combining ARQ system, thus they can improve the system reliability. Importantly, the effective throughput of the two schemes has a little improvement in the moderate SNR. In addition, we have also provided the result of power allocation. 


\section{Acknowledgement}

This work is supported by Major Research Plan of National Natural Science Foundation of China (No. 91438115), National Natural Science Foundation of China (No. 61371123, No. 61301165), Jiangsu Province Natural Science Foundation (BK2011002, BK2012055), Special financial grant of the China postdoctoral science foundation (2015T81079), China Postdoctoral Science Foundation (2014M552612), Jiangsu Postdoctoral Science Foundation (No.1401178C), Pre-research Project Foundation (9140C020306130C02007).

\section{References}

[1] I. Garcia, K. Sakaguchi, and K. Araki, "Cell planning for cooperative transmission," in Proc. IEEE WCNC'08, Las Vegas, USA, Mar. 2008, pp. 1769-1774.

[2] S. Moh, "Two cooperation models and their optimal routing for cooperative diversity in wireless ad hoc networks," in Proc. IEEE ISWCS'08, Reykjavik, Iceland, Oct. 2008, pp. 57-61.

[3] L. Sun, T. Zhang, L. Lu, and H. Niu, "Cooperative communications with relay selection in wireless sensor networks,” IEEE Trans. Consum. Electron., vol. 55, no. 2, pp. 513-517, May 2009.

[4] Y. Chen, J. Teo, J. Lai, E. Gunawan, K. S. Low, C. B. Soh, and P. Rapajic, "Cooperative communications in ultra-wideband wireless body area networks: Channel modeling and system diversity analysis,” IEEE J. Sel. Areas Commun., vol. 27, no. 1, pp. 5-16, Jan. 2009.

[5] S. Katti, D. Katabi, W. Hu, H. Rahul, and M. Medard, "The importance of being opportunistic: Practical network coding for wireless environments," in Proc. 43rd Allerton'05, Montecillo, Illinois, USA, Sep. 2005.

[6] S. Zhang, S. C. Liew, and P. P. Lam, "Hot topic: Physical-layer network coding," in Proc. 12th ACM MobiCom'06, New York, USA, Sep. 2006, pp. 358-365.

[7] S. Katti, S. Gollakota, and D. Katabi, "Embracing wireless interference: Analog network coding," in Proc. ACM SIGCOMM'07, Kyoto, Japan, Aug. 2007, pp. 397-408.

[8] S. Katti, H. Rahul, W. Hu, D. Katabi, M. Medard, and J. Crowcroft, "XORs in the air: Practical wireless network coding,” IEEE/ACM Trans. Netw., vol. 16, no. 3, pp. 497-510, Jun. 2008.

[9] Mohammad H. Amerimehr and Farid Ashtiani, "Delay and Throughput Analysis of a Two-Way Opportunistic Network Coding-Based Relay Network," IEEE Trans. Wirless. Comm, vol. 13, no. 5, May. 2014.

[10] J. Zhang, P. Fan, and K. B. Letaief, "Network coding for efficientmulticast routing in wireless ad-hoc networks,” IEEE Trans. Commun., vol. 56, no. 4, pp. 598-607, Apr. 2008.

[11] S. Katti, H. Rahul, W. Hu, D. Katabi, M. Medard, and J. Crowcroft, "XORs in the air: Practical wireless network coding," IEEE/ACM Trans. Netw., vol. 16, no. 3, pp. 497-510, Jun. 2008.

[12] Wang Xingwei, Wang Shiqiang, Song Qingyang \& Jamalipour, A. 2011. Power and rate adaptation for analog network coding. IEEE Trans. Veh. Technol. 60(5): 2302-2313.

[13] Z. Chen, C. Zhang, J. Zhang, and G. Wei, "ARQ protocols for two-way relay systems," in Proc. IEEE WiCOM, pp. 1-4, Sep. 2010.

[14] W. Guan and K. J. R. Liu, "On analysis of two-way relaying with networkcoded ARQ," in Proc. IEEE Globecom Workshops, pp. 4584-4589, Dec. 2013.

[15] Q.-T. Vien, L.-N. Tran, and H. Nguyen, "Network coding-based ARQ retransmission strategies for two-way wireless relay networks," in Proc. IEEE SoftCOM, pp. 180-184, Sep. 2010. 
[16] G. Yu, Z. Zhang, P. Qiu, "Efficient ARQ protocols for exploiting cooperative relaying in wireless sensor networks," Elsevier Journal of C omputer Communications, vol. 30, pp. 2765-2773, 2007.

[17] H. Liu, P. Popovski, E. de Carvalho, and Y. Zhao, "Sum-rate optimization in a two-way relay network with buffering," IEEE Commun. Lett., vol. 17, pp. 95-98, Jan. 2013.

[18] V. Jamali, N. Zlatanov, A. Ikhlef, and R. Schober, "Adaptive mode selection in bidirectional buffer-aided relay networks with fixed transmit bpowers," in Proc. EUSIPCO, pp. 1-5, Sep. 2013.

[19] Hataminia, S.; Vahidian, S.; Mohammadi, M.; Ahmadian-Attari, M., "Performance analysis of two-way decode-and-forward relaying in the presence of co-channel interferences," Communications, IET, vol.8, no.18, pp.3349,3356, 12182014.

[20] Z. Chen, C. Zhang, J. Zhang, and G. Wei, “ARQ Protocols for TwoWay Relay Systems," in Proc. IEEE WiCOM, Chengdu, China, Dec. 2010, pp. 1-4.

[21] S.-M. Cai and Y. Gong, "Cognitive beamforming for multiple secondary data streams with individual SNR constraints,” IEEE Trans. Signal Process., vol. 61, pp. 4189-4198, Sep. 2013.

[22] N. Sidiropoulos, T. Davidson, and Z.-Q. Luo, "Transmit beamforming for physical-layer multicasting,” IEEE Trans. Signal Process., vol. 54, pp. 2239-2251, Jun. 2006.

[23] J. F. Sturm, "Using SeDuMi 1.02, a MATLAB toolbox for optimization over symmetric cones," Optim. Meth. Softw., vol. 11-12, pp. 625-653, 1999. 